\title{
Alarma y alarmismo: medios de comunicación y crisis económica
}

\author{
Ángel ARRESE \\ Universidad de Navarra \\ aarrese@unav.es \\ Alfonso VARA-Miguel \\ Universidad de Navarra \\ avara@unav.es
}

Recibido: 15 de octubre de 2013

Aceptado: 27 de enero de 2014

\begin{abstract}
Resumen
La actual crisis económica mundial está generando buen número de estudios sobre su cobertura periodística. Una de las ideas más recurrentes es que en los años previos a la crisis los medios de comunicación fueron incapaces de alertar sobre los riegos que existían, y que después, ya durante la crisis, tienen una visión alarmista y simplificadora de la actualidad. En este artículo se hace un repaso y se valora la investigación realizada sobre estas dos acusaciones. La principal conclusión es que no existe consenso sobre la valoración de la actuación de los medios, ni antes de la crisis, ni después.
\end{abstract}

Palabras clave: crisis económica, crisis financiera, periodismo económico, cobertura mediática, crítica del sistema de medios

\section{"Alarm" and "alarmism": media coverage of the economic crisis}

\begin{abstract}
The current global financial and economic crisis is generating a good number of studies about its media coverage. One of the recurring ideas is that in the years before the crisis the media were unable to warn of the risks that exist in the financial system, and during the crisis, they are simplistic and alarmist. In this paper we review expert opinions and recent studies that address those two questions. The main conclusion of this paper is that there is no consensus on the assessment about media journalistic performance, before the crisis, or after.
\end{abstract}

Keywords: economic crisis, financial crisis, business journalism, media coverage, media criticism

\section{Referencia normalizada}

ARRESE, Ángel y VARA-MIGUEL, Alfonso (2014): “Alarma y alarmismo: medios de comunicación y crisis económica”. Estudios sobre el Mensaje Periodístico. Vol. 20, Núm. 2 (julio-diciembre), págs.: 933-951. Madrid, Servicio de Publicaciones de la Universidad Complutense.

Sumario: 1. Introducción. 2. Los medios y la crisis: ¿alarma insuficiente, o alarma excesiva?; 2.1. ¿Alertaron suficientemente los medios antes de la crisis?; 2.1.1. El juicio de los profesionales; 2.1.2. La crítica teórica; 2.2. ¿Crean excesiva alarma los medios durante la crisis?; 2.2.1. El juicio de los profesionales; 2.2.2. La crítica teórica; 2.2.3. Los resultados empíricos. 3. Conclusiones. 4. Referencias bibliográficas.

\section{Introducción}

«La prensa popular está llena de especulaciones sobre la posibilidad de que la burbuja inmobiliaria explote en Estados Unidos y en otros países. Barrons, la revista Money y The Economist han publicado diversos reportajes sobre el irracional rally de precios de las viviendas y sobre un potencial crash. The Economist ha publicado una serie de artículos con títulos como "Castles in Hot Air", "House of Cards", "Bubble Trouble" o "Betting the House". Estos relatos han generado lógicamente preocupación entre el público en general». (Case, K. E. y Shiller, R. J., 2003: 299). 
En los últimos cinco años, la crisis económica y financiera ha puesto en evidencia la importancia de los medios de comunicación en la generación y mantenimiento de estados de opinión económica, de los que es imposible disociar la evolución misma de la crisis. Aunque en la historia económica se han dado precedentes claros de esa interrelación entre medios y situación económica, sobre todo en momentos de boom y crash financieros (Arrese, 2010), quizá nunca como en la actualidad el análisis de esa interacción haya sido tan relevante.

En uno de los numerosos trabajos que se han publicado sobre las causas de la crisis financiera, The Financial Crisis. Who Is To Blame?, Howard Davis (2010) sintetiza las distintas narrativas que se han manejado en estos años, y destaca que una de ellas tiene que ver con la responsabilidad de los medios. Lo que plantea es que sobre todo en los años que precedieron a la crisis, los medios no fueron capaces de cumplir bien su función de "perros guardianes" en materia económica, y tampoco han cumplido posteriormente con su misión de alumbrar y explicar su complejidad. Esta línea de argumentación entronca con la versión más psicológica de la crisis, en la línea de los Animal Spirits de Akerlof y Shiller (2009), que da especial protagonismo a los estados de ánimo y a las historias que conforman la opinión pública en períodos de bonanza o de declive económico.

Otras investigaciones sobre la responsabilidad de distintas instituciones en la crisis han dejado completamente al margen la actuación de los medios. Sólo por citar dos ejemplos, en uno de los libros más alabados por su clarividencia al analizar las raíces del problema, Fault Lines, Rajan (2010) no dedica una sola línea a la idea de que los medios pudieran haber tenido algún papel en la gestación o en el desarrollo de la crisis. De igual forma, The Financial Crisis Inquiry Report (2011), la investigación sobre el tema comisionada por el gobierno estadounidense, en sus más de seiscientas páginas, no hace referencia alguna a la posibilidad de que los medios actuaran de forma irresponsable, o de que se mostraran incapaces de alertar sobre los riesgos económicos y financieros que existían en los años inmediatamente anteriores al desastre financiero.

Tampoco en España las reflexiones académicas sobre las causas y lecciones de la crisis, como la monografía de Fedea sobre La Crisis de la Economía Española. Lecciones y propuestas (2009), señalan a los medios como actores, por acción u omisión. En trabajos sobre aspectos más específicos de la crisis -como la burbuja inmobiliariatampoco se les responsabiliza. Al contrario, se utilizan algunas referencias mediáticas para demostrar que había sido una burbuja ampliamente anunciada (García Montalvo, 2008; Campos Echeverría, 2008).

Como en las publicaciones citadas, en la abundante bibliografía económica y financiera sobre las causas de la crisis existe un cierto consenso sobre las responsabilidades de ciertas instituciones, entre otras, entidades financieras, agencias de calificación, bancos centrales y gobiernos. También en muchos trabajos late la idea de que "toda la sociedad" tiene una cuota de culpabilidad en la generación de esta crisis sistémica (Davis, 2010; Mason, 2010). Pero por lo que se refiere a los medios, en los análisis expertos no queda nada claro si su papel ha sido el de una de esas instituciones decisivas -para bien o para mal-, o si simplemente forman parte de ese "toda la sociedad" más indefinido y genérico. 


\section{Los medios y la crisis: ¿alarma insuficiente, o alarma excesiva?}

La discusión sobre el papel de los medios en la actual crisis económica y financiera tiene muchas facetas, pero se puede sintetizar en dos grandes temas de debate. Por un lado, se cuestiona su actuación previa a la crisis, desde el punto de vista del cumplimiento o no de su función de alertar ante los riesgos y peligros que acechaban al sistema económico y financiero; por otro, se debate su comportamiento durante la crisis, en esta ocasión desde la óptica de si los medios generan o no una alarma excesiva en torno a las malas noticias, en lugar de explicarlas y contextualizarlas en su justa medida. O dicho de una forma más coloquial, utilizando las dos acepciones del verbo alarmar -avisar y asustar-, quienes critican la actuación de los medios a menudo senalan que no fueron suficientemente alarmistas antes de la crisis, y que lo han sido en exceso después. En palabras de Tulloch (2009), que han pasado de la "amnesia" al "apocalipsis".

\section{1. ¿Alertaron suficientemente los medios antes de la crisis?}

Es famosa la pregunta que la Reina Isabel II de Inglaterra hizo sobre la crisis financiera en una visita a la London School of Economics: “Cómo nadie la vio venir?" (Davis, 2010: 25). La Reina hacía esa pregunta a un grupo de economistas, pero bien podría estar pensando también en los medios. ¿Cómo los medios no la vieron venir?; o afinando algo más la pregunta -ya que prácticamente nadie previó la crisis tal y como se produjo-, ¿alertaron los medios suficientemente sobre los riegos financieros del sistema económico? Esta es la cuestión clave que entronca con el papel de los medios como parte del sistema de alertas necesario en una sociedad de riesgo como la actual (Beck, 1992).

\subsubsection{El juicio de los profesionales}

La variedad de testimonios en el mundo profesional sobre el papel preventivo de los medios es muy grande. Por ejemplo, los máximos responsables de dos publicaciones tan influyentes en las finanzas, como el Financial Times y el Wall Street Journal, mantienen versiones sensiblemente distintas sobre lo sucedido. Lionel Barber, director del diario británico, ha comentado en repetidas ocasiones que es indudable que los medios financieros pudieron haber hecho un mejor trabajo, del mismo modo que lo pudieron hacer antes del crash de las puntocom a principios de nuevo siglo (Barber, 2009); Marcus Brauchli, responsable editorial del periódico neoyorquino en la época previa a la crisis, defiende, por su parte, que hubo durante muchos años una intensa cobertura de los problemas y riesgos subyacentes del sistema financiero y de la economía. Según él, el periodismo en Estados Unidos envió abundantes señales de aviso (Strupp, 2008).

En línea con la primera idea, muchos periodistas han reconocido diversos problemas de los medios a la hora de identificar e investigar indicios de riesgo previos a la crisis: desde la falta de formación de los profesionales para comprender complejos productos financieros, hasta su ciega actitud reverencial ante los consensos oficiales, pasando por el facilón ensalzamiento de los mercados y sus protagonistas en los tiempos de bonanza (Caplen, 2009; Fuller, 2009; Harber, 2009). Asimismo, han vuelto a exponer los problemas organizativos, empresariales, etc. que tradicionalmente se citan 
para explicar la dificultad de una cobertura más profunda y crítica del mundo de los negocios (Kurtz, 2008; Stuller, 2008; Satija, 2009; Luscombe, 2009; Chittum, 2011).

Pero también hay quienes se resisten a aceptar sin más ni más el fracaso de los medios, o al menos la cuota de fracaso atribuida a lugares comunes más que discutibles. Usher (2012) describe esa resistencia entre los periodistas económicos del New York Times, y Francesco Guerrera, del Financial Times, niega la acusación de que haya existido un cierto "síndrome de Estocolmo" de la prensa financiera respecto a sus fuentes, anunciantes, etc. (Guerrera, 2009). Otros periodistas de renombre, como Larry Ingrassia, del New York Times, o Robert Peston, de la $B B C$, también han disculpado en buena medida a los medios, sobre todo por comparación con la actuación de otras instituciones (Sposito, 2009; Peston, 2009).

Casi de forma unánime, la mayoría de los profesionales que se han pronunciado en torno a este tema -tanto los críticos como los defensores de la actuación de los medioscree que por supuesto se pudo hacer más, sobre todo a la hora de conectar los indicios de riesgo que se advertían en distintos ámbitos del mercado, con las consecuencias que se produjeron posteriormente en el conjunto de la economía. Pero al mismo tiempo, se reconoce que los medios estaban en inferioridad de condiciones para realizar esa tarea respecto a analistas financieros, autoridades públicas o expertos, que casi sin excepción tampoco fueron capaces de advertir esas conexiones sistémicas.

En España ha existido poco debate profesional sobre este tema, aunque se han organizado varios seminarios profesionales, y los medios han dedicado algún reportaje a reflexionar sobre el periodismo de la crisis (véase, por ejemplo, Fernández, 2008a; Enguix, 2009; Pérez Oliva, 2012). Por lo que se refiere a la función de alarma previa a la crisis, lo cierto es que en nuestro país hubo una antelasa inmediata de la crisis, caracterizada por la pugna política y partidista, en la que -como señala García Montalvo- "es evidente que la prensa acertó más que el Gobierno cuando advirtió de la existencia de la crisis" (citado en, Enguix, 2009). Más discutible es el papel de los medios en torno a causas menos inmediatas, como la especulación inmobiliaria. Cuando algún destacado periodista económico se ha pronunciado sobre esa capacidad de previsión más de fondo, el juicio no ha sido muy positivo: "el periodismo no ha visto venir ninguna gran crisis económica" (Ekaizer, 2010).

En síntesis, no hay acuerdo claro sobre el modo en que los profesionales de los medios valoran su actuación previa a la crisis, aunque en términos generales se puede afirmar que la mayoría reconoce que pudieron hacer más para alertar sobre los peligros latentes del boom económico que la precedió. Al mismo tiempo, bastantes defienden que los medios, en comparación con otras instituciones implicadas, tuvieron un comportamiento más que digno.

\subsubsection{La crítica teórica}

Desde una perspectiva más teórica, o mejor, teniendo en cuenta reflexiones realizadas desde una óptica académica, se puede afirmar que la visión crítica casi ha monopolizado el juicio sobre el papel de los medios previo a la crisis. Son prácticamente unánimes los trabajos que dan por sentado que el periodismo económico no ha estado a la altura, debido a factores estructurales y profesionales. 
Entre los análisis estructurales, destacan aquellos que adoptan el enfoque de la economía política. Explican la incapacidad del periodismo económico para anticiparse a la crisis a partir de la naturaleza eminentemente capitalista del sistema de medios, condicionado por los intereses económicos de propietarios y anunciantes. A riesgo de simplificar, la idea de fondo es que en esas condiciones los medios son incapaces de adoptar una posición crítica ante el funcionamiento de la economía de mercado, y por tanto actuaron ciegamente como cheerleaders del boom que precedió a la crisis, sin advertir suficientemente sus enormes riesgos. En este sentido, sobre todo los medios especializados en economía y finanzas, son vistos como agentes clave del mercado, del sistema económico y financiero. Como tales, son partícipes en cada momento de su lógica de funcionamiento, y como consecuencia, están imbuidos de sus valores y principios. Además, tienen una posición débil respecto a otros agentes -inversores, empresas, etc.-, lo que dificulta todavía más y hace casi inviable el cumplimiento de su función de "perros guardianes".

Un ejemplo de este enfoque de análisis son algunos números especiales publicados sobre este tema en varias revistas académicas. Por ejemplo, el International Journal of Communication se plantea que la crisis no se puede entender como el misterioso fallo de ciertos componentes técnicos de la máquina económica del capitalismo, sino más bien como un fenómeno anclado en procesos informativos, culturales y mediáticos (Chakravartty y Downing, 2010: 695). Y desde ese punto de vista, por ejemplo, Chakravartly y Schiller (2010) defienden que "ciertos cambios en las prácticas del periodismo económico son un elemento clave en la actual crisis financiera" (p. 670), debido a la acrítica aceptación por parte de los medios del discurso económico neoliberal. Hope (2010), con una visión más macroscópica, explica la miopía de los medios financieros anglosajones en el contexto de su participación, como amplificadores de la incertidumbre financiera, en un capitalismo cada vez más financiarizado y necesitado de enormes flujos de información en tiempo real.

Entre los trabajos que tratan aspectos más profesionales que estructurales, quizá uno de los primeros que abordó los límites prácticos del periodismo económico en esta crisis fue el informe de Tambini (2008) titulado "What is Financial Journalism For?". En él se planteaba la necesidad de un modelo de derechos y obligaciones de los periodistas financieros, basado en el interés público, que superase el paradigma actual, centrado en los intereses informativos de los mercados y de los inversores. En un trabajo posterior, Tambini (2010) afirmaba que para comprender "por qué los medios prestaron tan poca atención a los riegos del sistema bancario en la antesala de la crisis financiera, es necesario comprender el marco de leyes, regulaciones, autorregulaciones e incentivos profesionales que estructura la práctica del periodismo económico" (p. 158). En cierta medida, este enfoque seguía la línea de investigaciones precedentes, como las de Davis (2005) o Doyle (2006), que ponen el acento de los fallos del periodismo económico en las especiales circunstancias del ejercicio profesional en ese campo, desde las carencias de formación hasta los fuertes de desequilibrios en la relación con las fuentes, sobre todo en información financiera. Meier y Winterbauer (2008) hacen un análisis parecido, a partir de entrevistas con profesionales, para el caso del periodismo económico alemán. Usher (2012), por su parte, se centra en el 
caso estadounidense, poniendo énfasis en la escasa mentalidad de periodismo de "perro guardián" que existe en las redacciones económicas.

Otros autores, en la misma línea de trabajo que los anteriores, destacan causas diversas de la ceguera de los medios en los años previos a la crisis: Fraser (2009) y Schechter (2009) inciden en aspectos profesionales y de limitación de recursos redaccionales; Manning (2012) y Davis (2011), por su parte, ponen el énfasis en las relaciones entre medios y fuentes expertas, sobre todo financieras, en contextos de generalizada aceptación de la "sabiduría recibida".

Como sumario de este apartado, se puede afirmar que el mundo académico, casi por unanimidad, se ha alineado con la idea de que el comportamiento del periodismo económico previo a la crisis fue deficiente o muy deficiente. Seguramente la mayoría de los autores están de acuerdo con la sentencia de Fray (2011), en uno de los artículos del número especial sobre el tema de la British Journalism Review: "la verdad es que cuando los negocios y las finanzas se convirtieron en la mejor historia del mundo, a casi todos los periodistas se les pasó" (Fray, 2011: 53).

\subsubsection{Los resultados empíricos}

Los estudios empíricos sobre el papel de los medios en la antesala de la crisis no son muy numerosos. Y los que existen -generalmente análisis de contenidos- presentan resultados contradictorios o llegan a conclusiones dispares. Además, tanto desde el punto de vista metodológico, como del objeto de estudio, es difícil compararlos.

En Estados Unidos los dos estudios más comentados han sido los de Chris Roush (2008) y Dean Starkman (2009). Ambos se publicaron en dos revistas semiacadémicas con gran prestigio profesional, la American Journalism Review y la Columbia Journalism Review, respectivamente.

En el primer trabajo, "Unheeding Warnings", Roush, profesor de periodismo económico en la Universidad de North Carolina (Chapel Hill), llega a la conclusión de que los principales medios periodísticos alertaron suficientemente a la población sobre ciertos riesgos financieros. Pero, según Roush, la gente, en general, no quiso conceder demasiado crédito a esas alarmas, tesis que ha seguido mantenido en reflexiones posteriores, tras el debate surgido en torno a su análisis (Roush, 2011). Por su parte, Starkman, antiguo periodista del Wall Street Journal y director de la sección "The Audit" de la Columbia Journalism Review, critica abiertamente en el artículo "Power Problem" las conclusiones de Roush, a partir del análisis de una muestra de 730 artículos publicados entre 2000 y 2007 en algunos de los medios más influyentes en el ámbito de la información económica estadounidense (The Wall Street Journal, The New York Times, Los Angeles Times, The Washington Post, Bloomberg News, Financial Times, Fortune, Business Week, y Forbes). Según este autor, si bien en el período 2000-2003 hubo un notable número de investigaciones periodísticas que denunciaron ciertos problemas y riesgos del sistema financiero, especialmente la conexión entre Wall Street y el mercado hipotecario, en los años inmediatamente anteriores a la crisis, entre 2003 y 2006, ese tipo de trabajos casi desaparecieron. En consecuencia, según Starkman, no se puede decir que los medios cumplieran su función de "perros guardianes", precisamente en ese período clave. 
Esos dos trabajos han polarizado la discusión profesional sobre este tema en Estados Unidos, pero poco a poco van apareciendo más estudios que los complementan. Es el caso de un trabajo reciente de Chernomas y Hudson (2011) sobre la historia de la cobertura económica del New York Times, que también dedica gran atención a la actuación del diario neoyorquino en torno a la crisis. Los autores concluyen que el periódico realizó un trabajo más que aceptable durante los años previos a la crisis, en especial por su tratamiento crítico del fenómeno de la desregulación financiera, resultado de los estrechos vínculos entre las finanzas y la política. Lo que no hizo el Times, concluyen, fue "extender sus análisis más allá de los problemas y fracasos concretos de los sectores inmobiliario, bancario y financiero, para ponerlos en relación con los temas más generales del estancamiento de los salarios y la creciente desigualdad que acabó mermando el consumo de los hogares estadounidenses" (p. 165).

Quizá sea en Alemania donde se cuente con una investigación empírica más solvente sobre este tema, en especial por un cuidado trabajo de Arlt y Storz (2010). En la monografia "Wirtschaftsjournalismus in der Krise" se analiza la cobertura periodística de cinco periódicos de calidad (Frankfurter Allgemeine Zeitung, Financial Times Deutschland, Handelsblatt, Süddeustsche Zeitung y Tageszeitung). Su conclusión sobre la actuación de estos medios en el período 1999-2005 es contundente: en general, se dejaron llevar por la exaltación del funcionamiento cada vez más libre y desregulado de los mercados financieros, y prácticamente no advirtieron riesgos de calado para el sistema económico. De todos ellos, sólo el Financial Times Deutschald mostró una sensibilidad crítica algo mayor, pero ninguno escapaba a la calificación de "mal" o "muy mal" con que los autores valoran su capacidad previsora. Algo más positivo es el resultado del análisis de contenidos llevado a cabo por Schranz y Eisenegger (2011), en el que se compara la cobertura de la crisis llevada a cabo por tres diarios de gran prestigio internacional (el suizo Neue Zürcher Zeitung, el británico The Guardian y el estadounidense New York Times).

Los trabajos que han afrontado este tema en España son escasos, aunque hay algunas investigaciones en marcha. Es el caso del proyecto doctoral iniciado por Stefanie C. Müller, corresponsal en España del semanario alemán Wirstchaftswoche. En su trabajo de investigación El papel de la prensa en la mayor crisis económica que ha vivido la democracia española, Müller (2010) estudia las portadas de El País y El Mundo entre 1996 y 2009, y llega a la conclusión de que en ese tiempo "los dos diarios no cumplieron con su deber de informar sobre acontecimientos de manera objetiva, analítica e informativa" (p. 101). Más en concreto, indica que "los medios españoles han jugado un papel importante en la prolongación del fenómeno especulativo en el sector inmobiliario (p. 2). Un reciente estudio de Arrese y Vara (2012), sin embargo, matiza algo ese juicio, al demostrar que la discusión sobre un concepto tan cargado de significado y de referencia al riesgo, como el de "burbuja inmobiliaria", fue bastante común en los principales diarios entre 2003 y 2008, a pesar de la insistencia, por parte de expertos y de fuentes gubernamentales, en la idea de que dicha burbuja no existía.

Como resumen de este apartado, no se puede afirmar de forma concluyente que la investigación empírica demuestre la ceguera de los medios ante los fenómenos que, 
a la postre, se demostrarían como centrales en la explicación de las cusas de la crisis. Más bien, parece que hay diversidad de situaciones, dependiendo de países, medios de comunicación y temas concretos.

\section{2. ¿Crean excesiva alarma los medios durante la crisis?}

En 2009 la Comisión del Tesoro del Parlamento Británico abrió una investigación para analizar la actuación de los medios durante la crisis, y más específicamente, si las informaciones de Robert Peston en la BBC habían podido desencadenar el pánico bancario que acabó con la quiebra de Northern Rock (UK Parliament Treasury Committee, 2009). De fondo, latía la idea de poder plantear que en momentos de crisis financiera se restringiera o supervisara la actuación del periodismo económico para evitar la difusión de informaciones y rumores capaces de generar pánico en los mercados. A grandes rasgos, la Comisión exoneró de responsabilidad a los medios en ese pánico financiero.

Pero el problema no sólo son los mercados financieros. El tono de las noticias, la visión más o menos pesimista de la actualidad, la utilización de simplificaciones y estereotipos, etc. adquieren especial relevancia en momentos de crisis económica. En tal situación, además de las ya tradicionales tensiones que caracterizan al ejercicio del periodismo económico (Arrese, 2006), los medios deben afrontar el hecho de que sus contenidos generan estados de opinión, niveles de confianza o desconfianza, que inciden directamente en la propia marcha de la economía.

En España esta realidad se ha vivido de forma especialmente intensa. Cabe recordar la reunión del presidente Zapatero con los editores de los principales grupos de prensa, para pedirles que evitasen la crítica alarmista y que trataran de infundir tranquilidad a los ciudadanos, sobre todo en torno a la solvencia del sistema financiero (González y Lobo, 2008). Se inscribe también aquí la tensa relación con los medios internacionales, acusados en ciertos momentos de atacar sin fundamento a la economía española, lo que incluso llevó a que se pidiera al Centro Nacional de Inteligencia que investigara el rol de la prensa internacional anglosajona en el fomento de la crisis de la deuda soberana (Tremlett, 2010; Cendrowicz, 2010; Der Spiegel, 2010; The Economist, 2010).

Frente a la acusación genérica de alarmismo de los medios, también hay autores que critican lo contrario: que los medios, durante la crisis, son excesivamente cautos, y se contagian con facilidad de las directrices de las autoridades, interesadas en no alarmar en exceso a la población y a los mercados. Otros simplemente reconocen que, al menos durante esta crisis, los medios en general han hecho un trabajo más que razonable para explicar lo que realmente sucede; un trabajo que por otra parte no es nada fácil, teniendo en cuenta la complejidad de la situación y las muchas posturas no siempre coincidentes que mantienen sobre ella los expertos, los organismos internacionales, gobiernos, etc.

\subsubsection{El juicio de los profesionales}

La crisis todavía no ha terminado y no abundan los testimonios de los propios profesionales sobre su desempeño durante estos años. En general se reconoce que los me- 
dios han hecho un enorme esfuerzo para dedicar espacio y tiempo a la actualidad económica, convertida de la noche a la mañana en la estrella informativa de las redacciones (Fernández, 2008a). Incluso en medios ya especializados, como el Financial Times, se reorganizaron los contenidos del diario para dedicar casi el $50 \%$ de sus páginas a temas relacionados con la crisis (Thompson, 2008).

Por lo que respecta al alarmismo, la mayoría de los profesionales piensan que se dan excesos de forma puntual, pero que, en general, los periodistas y los medios que tratan de hacer buena información económica son muy conscientes de la sensibilidad de la información que manejan. En Estados Unidos, cabeceras como el Wall Street Journal, New York Times, Washington Post, o Fortune -por citar algunas de las más conocidas- fueron muy escrupulosas con la utilización de términos y adjetivos extremos para describir ciertos momentos difíciles, sobre todo al principio de la crisis (Pérez-Peña, 2008). En Gran Bretaña, la gran mayoría de los testimonios profesionales que se escucharon en la ya citada Comisión del Tesoro del Parlamento también iban en la misma dirección. La declaración de la Newspaper Society, en representación de toda la prensa local y regional británica, concluía:

"No hay evidencia alguna que los medios hayan cubierto la presente crisis financiera de forma poco profesional y defectuosa. De hecho, la única crítica -que es la génesis de la posible idea de imponerles restricciones- parece ser que han sido demasiado rigurosos y acertados al hacerlo" (UK Treasury Committee, 2009).

Tampoco en España se percibe un particular mea culpa profesional en relación al sensacionalismo. Magis Iglesias, presidenta de la Asociación de Periodistas de España, comentaba: "La cobertura que se está haciendo en España sobre la crisis económica está siendo buena. Es evidente que hay algunos aprovechados que abrazan el amarillismo para vender más periódicos, pero son siempre casos aislados" (citado en Fernández, 2008b). Por su parte, Joaquín Estefanía, veterano periodista económico, afirmaba recientemente: "Los medios de comunicación y los periodistas debemos movernos en este asunto en unos límites muy estrechos: por una parte debemos contar y explicar lo que sucede, por muy grave y alarmante que sea (y la actual crisis económica es muy profunda, muy larga y está dejando unas secuelas terribles en la sociedad española), y por otra, debemos evitar inocular el miedo a los ciudadanos que nos leen, nos escuchan o nos ven" (citado en Pérez Oliva, 2012).

Donde existe más disparidad de opinión es entre expertos que colaboran habitualmente con los medios, aunque en general se han oído más críticas que alabanzas. Para algunos, no ha habido excesivo alarmismo y catastrofismo, sino más bien el realismo que requería el difícil momento por el que atravesaba España, como señala García Montalvo (citado en, Enguix, 2009); para otros, como el sociólogo Gil Calvo, los medios han sido juez y parte en el relato de la crisis, y son "culpables de interferencia interesada y distorsionadora" (citado en, Pérez Oliva, 2012). Martínez Abascal ha criticado el enfoque negativo de las noticias: "Los medios de comunicación dan mucho más énfasis a las noticias negativas que a las positivas. Lo negativo vende. Es más, lo que realmente vende no es la noticia de algo negativo que ha pasado, sino pronosticar grandes desastres futuros y próximos” (Martínez Abascal, 2011). 
En otros países con una situación económica también muy complicada, como por ejemplo Irlanda, se confirma la idea de que los periodistas económicos desplegaron en la cobertura de la crisis mayor decisión y profesionalidad que en años previos (Fahy, O’Brien y Poti, 2010).

En resumen, se puede afirmar que el juicio genérico de los profesionales sobre la cobertura de la crisis tiende a ser positivo, mucho más que el existente respecto a la capacidad predictiva de los medios antes de que se iniciara. Menos positiva parece ser la opinión de los expertos que participan en la discusión de estos temas en la opinión pública, que acostumbran a llamar la atención sobre los aspectos menos afortunados y criticables de la cobertura periodística. En conjunto, como señala Schiffrin, quizá se podría coincidir en que "la cobertura no ha sido mala, aunque tampoco especialmente innovadora y prospectiva" (Schiffrin, 2011b).

\subsubsection{La crítica teórica}

En el plano teórico no se hace una especial distinción en la crítica a los medios por su actuación antes o después de la crisis. Prácticamente todos los argumentos utilizados para explicar su fracaso como alertas de la crisis son válidos también para argumentar sobre la deficiente cobertura, una vez que aquella estalló. Y por tanto, predomina con claridad la idea de que los medios son incapaces de abordar adecuadamente una situación de crisis como la actual.

Un ejemplo de esta visión es el monográfico de la revista TripleC, títulado "Capitalist Crisis, Communication \& Culture". Aunque las referencias al papel de los medios en la crisis no son muy abundantes, las que se hacen van en la dirección ya comentada. En uno de los artículos, por ejemplo, de Mateo y Berges (2010), tomando como referencia el caso de España, señalan que la falta de independencia económica de los medios, muy condicionados por sus obligaciones financieras, ha hecho que durante esta crisis sea difícil encontrar artículos de calidad y análisis profundos sobre los problemas de los bancos y del sistema financiero. Ese enfoque corporativo es también el eje de las críticas lanzadas en otros foros por autores como Almirón (2008) y Lits (2010).

Siguiendo con esa visión estructural, hay otras propuestas de explicación de la deficiente cobertura de la crisis, como por ejemplo la de Stiglitz (2011), desde la economía de la información y de los incentivos que existen en el mercado para distorsionar los mensajes, o la de Thompson (2009), aplicando el modelo de propaganda de Herman y Chomsky a la producción y difusión de noticias financieras. Asimismo, varios autores han criticado la creciente "politización" del tratamiento periodístico, que ha llevado a los medios a dejar a un lado análisis e interpretaciones bien fundamentadas, para acabar alineándose con visiones partidistas de la situación (Becerra y Mastrini, 2010; Caruso, Cepernich y Rocarolo, 2012).

Por último, desde enfoques más culturales, también se han analizado otros síntomas de la dificultad de los medios para salirse del discurso oficial y del marco de análisis del pensamiento capitalista. Así, por ejemplo, Sandvoss (2010) sostiene que el periodismo económico y financiero, inserto en las estructuras de corporaciones mediáticas transnacionales y del capitalismo global, no puede sino reproducir en sus dis- 
cursos los intereses de ese sistema. Eso hace que, por ejemplo, en los medios se hable del "mercado de las hipotecas subprime" (poniendo énfasis en la incapacidad de ciertos consumidores para hacer frente a sus deudas) en lugar de hablar del "préstamo predatorio" (que pondría énfasis en la falta de responsabilidad y prudencia de la banca al conceder esas hipotecas). En la misma línea de análisis del discurso de los medios, Lewis (2010) explica cómo la aceptación sin reservas del marco conceptual que dice que el "crecimiento es bueno", llevó a los medios a no plantearse dudas o críticas serias en torno a las grietas del sistema económico.

De nuevo, casi sin excepción, los análisis teóricos enmarcan lo que consideran una deficiente cobertura de la crisis en las mismas coordenadas de crítica utilizadas para entender la incapacidad de los medios para anticiparla.

\subsubsection{Los resultados empíricos}

Poco a poco se empiezan a publicar trabajos empíricos sobre distintos aspectos del tratamiento de la crisis. Aunque todavía hay mucha heterogeneidad en el tipo de estudios, $\mathrm{y}$ en los enfoques de investigación, se pueden exponer algunas de las ideas generales que se deducen de ellos.

Por lo que se refiere a juicios generales sobre la cobertura, no se puede decir que las investigaciones existentes lleguen a conclusiones claras. Schiffrin y Fagan (2012), tras analizar en 16 grandes medios la cobertura del paquete de estímulo económico estadounidense de 2009, señalan que estos no jugaron un papel importante en la fijación de la agenda y fueron a remolque de los temas planteados por las fuentes gubernamentales. Marron (2010), en su investigación sobre los medios británicos e irlandeses, resalta que la mayoría se hicieron eco de las noticias coyunturales que afectaban a las instituciones financieras nacionales e internacionales, pero fueron incapaces de ponerlas en relación con la crisis sistémica del crédito que se desencadenó posteriormente. Hicieron un excelente trabajo en la cobertura de la actualidad, pero la nota fue muy deficiente en el análisis de sus implicaciones.

Como contraste a estos resultados más bien negativos, Quring y Weber (2012) llegan a la conclusión de que la cobertura en medios alemanes de las medidas anticrisis del gobierno fue "ponderada y equilibrada", juicio especialmente positivo si tiene en cuenta que su análisis se centra en las noticias televisivas. Por su parte, Schifferes y Coulter (2012), en referencia al estudio de la cobertura online de la $B B C$, resaltan que ésta no fue tan superficial como a veces se supone.

Otros aspectos más particulares del tratamiento de la crisis, conectados con críticas tradicionales al periodismo económico, son objeto de evidencias más claras. Por ejemplo, Bähr (2009) ha expuesto el predominio de las noticias negativas y de las visiones pesimistas sobre la crisis en la prensa alemana, un hallazgo que por otra parte ya se había verificado también en las noticias económicas en épocas sin crisis (Lowry, 2008). Tracy (2012) ha denunciado la estereotipada y simplista visión que de la crisis griega y de sus razones han dado los medios estadounidenses, al centrarse en aspectos culturales y de idiosincrasia de los griegos, dejando a un lado otro tipo de explicaciones más institucionales, que implican a otros protagonistas europeos y estadounidenses. Desde un enfoque más lingüístico, Lits (2008), en su análisis de ocho 
diarios y revistas francesas, constata la excesiva utilización de términos dramáticos y que apelan a las emociones. Y Horner (2011), en Estados Unidos, critica el abuso de metáforas cargadas de contenido ideológico, tanto en los medios que estaban a favor como en los que estaban en contra del rescate de Wall Street en 2008.

Un último campo de estudio es el del predominio o no de fuentes y marcos de análisis oficiales, frente a la posibilidad de dar protagonismo a discursos alternativos. En este sentido, Schranz y Eisenegger (2011), en el estudio ya comentado, concluyen que en ningún momento los medios más prestigiosos presentan alternativas al orden capitalista imperante, aunque en muchos casos sean muy críticos con sectores como el financiero. A similar conclusión llega Mylonas (2012) al analizar la cobertura del diario popular alemán Bild Zeitung, Berry (2012), al hacerlo con el tratamiento de la crisis bancaria en el programa Today de la BBC, o Titley (2012) al estudiar iniciativas periodísticas alternativas surgidas en Irlanda para responder ante la acrítica aceptación por parte los medios del discurso oficial del inevitable rescate del país. En todos estos estudios, como en otros más específicos sobre la utilización de fuentes expertas (véase, por ejemplo, Sarabia-Panol y Sison, 2010), se pone de manifiesto el muy limitado abanico de voces que se utiliza para explicar e interpretar la actualidad, la mayoría de ellas representantes del sistema político, económico y financiero.

Así pues, la investigación empírica también ofrece resultados dispares sobre la valoración de la cobertura de la crisis por parte de los medios, aunque hay que reconocer que existen más estudios sobre aspectos mejorables de esa cobertura, que sobre la justificación de su calidad.

\section{Conclusiones}

El repaso de la investigación relativa al papel de los medios en la crisis financiera, así como de las reflexiones sobre el grado en que alertaron o no de los riesgos del sistema en los años previos, no permite sacar conclusiones claras al respecto. La variedad metodológica, las distintas formas de enfocar el objeto de estudio -la crisis, alguno de sus aspectos concretos, sus causas, responsables-, la diversidad de situaciones vividas en diferentes países, la heterogeneidad de juicios profesionales, etc. dificultan dar respuesta clara a ambas cuestiones.

Existe bastante consenso al afirmar que los medios, sobre todo los más influyentes en este ámbito informativo -prensa de calidad y especializada-, pudieron haber cumplido mejor su función de "canarios en la mina" en los años previos a 2008. Pero en eso, como ya se ha comentado, no han sido muy distintos a muchas otras instituciones -gobiernos, autoridades económicas, organismos internacionales, agencias de calificación, banca, etc.- cuya actuación pública fue al menos tan mejorable, si no más.

Asimismo, parece aceptarse que iniciada ya la crisis la conciencia de la importancia de mejorar la cobertura, y de cuidar el impacto de las noticias en la economía, aumentó en la mayoría de las redacciones. Pero tampoco los medios se han podido despegar del todo de los factores estructurales y profesionales que condicionan su cobertura, y que los hacen en exceso dependientes de las fuentes expertas y de las visiones oficiales de la realidad, en general inmersas en el marco de análisis de la economía capitalista. 
En general, la visión de los profesionales es más bien positiva sobre el desempeño de los medios en esta crisis, aun reconociendo que se pudo hacer más y mejor, sobre todo en los años que precedieron al colapso financiero; por el contrario, desde el mundo académico se dibuja un panorama muy negativo, de un periodismo económico incapaz de desempeñar su función de servicio a la sociedad, atrapado en una red de intereses empresariales y de condicionantes profesionales que lo atenazan. La investigación empírica, por su parte, empieza a ofrecer resultados mixtos, tanto de actuaciones y prácticas dignas de alabanza -en la cobertura en general, o en aspectos particulares-, como de problemas que se han puesto en evidencia con claridad, muchos de ellos confirmando algunas de las críticas históricas al periodismo económico.

Quizá, como reflejo de esta difícil evaluación del papel de los medios en la crisis, en una reciente investigación de Schifferes (2012) sobre la opinión de los británicos en torno a este tema, la mitad de la población estaba satisfecha con la cobertura de la crisis, y la otra mitad, insatisfecha. Mucha gente mostraba también su desconfianza respecto a la objetividad del periodismo económico, a su dependencia de fuentes muy parciales y su incapacidad para hacerse cargo de las preocupaciones de la gente de la calle.

La crisis no ha concluido y será necesaria más perspectiva para hacer una valoración conjunta del papel de los medios de comunicación en estos años. Mientras tanto, es importante seguir aportando evidencias sobre el grado en que el periodismo económico cumple y ha cumplido o no con su función, en un momento tan importante de la historia económica.

\section{Referencias bibliográficas}

AKERLOF, George \& SHILLER, Robert J. (2009): Animal Spirits. How Human Psychology Drives the Economy, and Why It Matters for Global Capitalism. Princeton: Princeton University Press.

ALMIRON, Nuria (2008): "Crisis financiera, economía y medios de comunicación", en Mientras Tanto, vol. 108-109, pp. 83-90.

ARLT, Hans-Jrgen \& STORZ, Wolfgang (2010): Wirtschaftsjournalismus in der Krise. Frankfurt/Main, Otto Brenner Stiftung.

ARRESE, Ángel (2006): "Simplificación y rigor en el periodismo económico", en Cuadernos de Información, 19, pp. 42-49.

ARRESE, Ángel (2010): "Periodismo económico, entre el boom y el crash", en ARRESE, Ángel et al. (coords.). Periodismo económico. Viejos y nuevos desafios. Actas del XXII Congreso Internacional de Comunicación (Periodismo económico. Viejos y nuevos desafios) (pp. 17-27). Pamplona, Eunsa.

ARRESE, Ángel y VARA, Alfonso (2012): “¿Canarios en la mina? La prensa y los riesgos de la "burbuja inmobiliaria" en España", en "Comunicación y riesgo". III Congreso Internacional de la Asociación Española de Investigación en Comunicación, AE-IC, Tarragona, 20 de diciembre de 2012.

BÄHR, Helena (2009): "The world economic crisis and its actors in the on-line publications of German newspapers and journals", en Journal of Media Research, 4, 56-64. 
BARBER, Lionel (2009): "Did financial journalists miss the financial crisis?", en Poynter Fellowship Lecture, Yale University, April 21. http://cjrarchive.org/img /posts/LionelBarber-lecture.pdf. [consultado el 8/08/11]

BECERRA, Martin Alfredo y MASTRINI, Guillermo (2010): "Crisis. What Crisis? Argentive Media in View of the 2008 International Financial Crisis", en International Journal of Communication, 4, 611-629.

BECK, Urlich (1992): The Risk Society. London, Sage.

BENNETT, Philip (2011): "When a Crisis in Journalism Meets an Economy in Crisis", en History of Political Economy, 43 (2), 375-378.

BERRY, Mike (2012): "The Today programme and the banking crisis", en Journalism, Online First Version, 27 September, 2012.

BRUMMER, Alex (2009): "Far from scaring people, the press were providing readers with reliable information", en Ethical Space: The International Journal of Communication Ethics, 6 (3/4), Special Issue, 38-41.

CABRALES, Antonio; DOLADO, Juan J.; FELGUEROSO, Florentino; y VÁZQUEZ, Pablo (coords., 2009): La Crisis de la Economía Española. Lecciones y propuestas. Madrid, Sociedad Abierta-Fedea.

CAMPOS ECHEVERRÍA, José Luis (2008): La burbuja inmobiliaria española. Madrid, Marcial Pons.

CAPLEN, Brian (2009): "Mea culpa: Why we missed the crisis", en Ethical Space: The International Journal of Communication Ethics, 6 (3/4), Special Issue, pp. 28-32.

CARUSO, Loris; CEPERNICH, Cristopher; e ROCAROLO, Francesco (2012): “La rappresentazioni mediali della crisi tra bisogni informativi e strategie político-comunicative", en Rassegna Italiana di Sociologia, 1, 137-168.

CASE, Karl E. \& SHILLER, Robert J. (2003): "Is There a Bubble in the Housing Market?", en Brookings Papers on Economic Activity, 2, pp. 299-362.

CENDROWICZ, Leo (2010): "Conspiracists Blame Anglo-Saxons, Others for Euro Crisis", en Time, 26 February.

CHAKRAVARTTY, Paula \& SCHILLER, Dan (2010): "Neoliberal Newspeak and Digital Capitalism in Crisis". International Journal of Communication, 4, pp. 670692.

CHAKRAVARTTY, Paula \& DOWNING, John D. (2010): "Media Technology and the Global Financial Crisis". International Journal of Communication, 4, pp. 693695.

CHERNOMAS, Robert y HUDSON, Ian (2011): The Gatekeeper. 60 Years of Economics According to the New York Times. Boulder, Paradigm Publishers.

CHITTUM, Ryan (2011): "Missing the Moment”, en Anya SCHIFFRIN: Bad News. How America's Business Press Missed the Story of the Century (pp. 71-93).New York, The New Press. 
DAVIES, Howard (2010): The Financial Crisis. Who is to Blame? London, Polity Press.

DAVIS, Aeron (2005): "Media Effects and the Active Elite Audience: A Study of Media in Financial Markets", en European Journal of Communication, 20 (3), pp. 303-326.

DAVIS, Aeron (2011): "News of the Financial Sector: reporting on the City or to it?", en Open Democracy, 31 May, en: http://www.opendemocracy.net/ourkingdom /aeron-davis/news-of-financial-sector-reporting-on-city-or-to-it

DE MATEO, Rosario; BERGÉS, Laura; y GARNATXE, Anna (2010): "Crisis, what crisis? The media: business and journalism in times of crisis", en Triple C. Cognition, Communication, Co-Operation, 8 (2), pp. 193-204.

DER SPIEGEL (2010): “A Media Plot against Madrid?: Spanish Intelligence Reportedly Probing 'Attacks' on Economy”, 15 February.

DOYLE, Gillian (2006): “Financial News Journalism”, en Journalism, 7 (4), pp. 433 452.

ENGUIX, Salvador (2009): "Prensa veraz contra el pánico. Los expertos valoran el papel que el periodismo ha desempeñado en esta crisis", en La Vanguardia, 7 de octubre.

EKAIZER, Ernesto (2010): “El periodismo ‘empotrado' y la crisis”. Público, 22 de noviembre.

FAHY, Declan; O'BRIEN, Michael; \& POTI, Valerio (2010): “Combative Critics or Captured Collaborators? Irish Financial Journalism and the End of the Celtic Tiger", en Irish Communications Review, 12, 5-21.

FERNÁNDEZ, Ángel (2008a): "La crisis se populariza en los medios", en El Mundo, 27 de octubre.

FERNÁNDEZ, David (2008b): "Callar, nunca", en El País, 24 de diciembre.

FERNÁNDEZ VILLAVERDE, Jesús (2010): "Nada Es Gratis cumple un año”, en Nada es Gratis, 16 de junio. http://www.fedeablogs.net/economia/?p=4855

FRASER, Matthew (2009): "Five reasons for crash blindness", en British Journalism Review, 20 (4), pp. 78-83

FRAY, Stephen (2011): “Big City, bright lights”, en British Journalism Review, 22 (1), pp. 48-53.

FULLER, Jane (2009): "Why journalists need to relearn the old habits of scepticism, fearless questioning and digging for information", en Ethical Space: The International Journal of Communication Ethics, 6 (3/4), Special Issue, pp. 87-91.

GARCÍA MONTALVO, Javier (2008): De la quimera inmobiliaria al colapso financiero. Crónica de un desenlace anunciado. Barcelona, Antonio Bosch.

GONZÁLEZ, A. y LOBO, José Luis (2008): "Pacto de silencio: los grandes editores de prensa se comprometen a apoyar a Zapatero ante la crisis", en El Confidencial, 
16 de octubre. http://www.elconfidencial.com/cache/2008/10/16/comunicacion_33_pacto_silencio_grandes_editores_prensa_comprometen_apoyr.html [consultado el 8/01/10]

GUERRERA, Francesco (2009): "Why generalists were not equipped to cover the complexities of the crisis", en Ethical Space: The International Journal of Communication Ethics, 6 (3-4), Special Issue, pp. 43-49.

HARBER, Anton (2009): “When a watch dog doesn't bark”, en Rhodes Journalism Review, 29 (1), pp. 20-21.

HOPE, Wayne (2010): “Time, Communication and Financial Collapse”, en International Journal of Communication, 4, 649-669.

HORNER, Jennifer (2011): "Clogged systems and toxic assets. News metaphors, neoliberal ideology, and the United States 'Wall Street Bailout' of 2008", en Journal of Language and Politics, 10 (1), 24-49.

KRUGMAN, Paul (2009): "How Did Economists Get It So Wrong?", en The New York Times, September 6.

KURTZ, Howard (2008): "Press May Own a Share in Financial Mess", en The Washington Post, October 6.

LEWIS, Justin (2010): "Normal Viewing Will Resumed Shortly: News, Recession and the Politics of Growth", en Popular Culture, 8, pp. 161-165.

LITS, Marc (2008): "Les médias face à la crise", en Médiatiques, 43, 3-7.

LITS, Marc (2010): "Pourquoi les médias n'ont-ils rien vu venir?", en DUJARDIN, Vincent; DE CORDT, Yves; COSTA, Rafael; et DE MORIAMÉ, Virginie de (dir.): La crise économique et financière de 2008-2009: l'entrée dans le 2le siècle? (pp. 81-94). Paris, Peter Lang.

LOWRY, Dennis T. (2008): "Network TV News Framing of Good vs. Bad Economic News under Democrat and Republican Presidents: A Lexical Analysis of Political Bias", en Journalism \& Mass Communication Quarterly, 85 (3), 2008, 483-498.

LUSCOMBE, Belinda (2009): "Business Journalism: A Vanishing Necessity?”, enTime, July 20.

MANNING, Paul (2012): "Financial Journalism, news sources and the banking crisis", en Journalism (Online First Version, May 29, 2012).

MARRON, María B. (2010): "British/Irish Media Excel in Episodic Coverage, Fail in Probing", en Journalism Studies, 11 (2), pp. 270-274.

MARTÍNEZ ABASCAL, Eduardo (2011): "Cuidado con las noticias catastróficas", en Expansión, 19 de enero.

MASON, Paul (2010): Meltdown: The End of the Age of Greed. London, Verso.

MEIER, Cristian \& WINTERBAUER, Stefan (2008): Die Finanzkrise und die Medien: Nagelprobe für den Wirtschafts- und Finanzjournalismus, Dossier. MainzerMedienDisput, November. 
MÜLLER, Stefanie Claudia (2010): El papel de la prensa en la mayor crisis económica que ha vivido la democracia española. DEA, Universidad Complutense de Madrid, 2010. http://www.scm-communication.com/files/dea16.pdf [consultado el 5/03/11].

MÜLLER, Stefanie Claudia (2011): "The Real State Bubble in Spain has been Pumped Up by All of Us", en Aestimatio. The IEB International Journal of Finance, 2, pp. 2-11.

MYERS, David S. (1982), "Editorials on the Economy in the 1980 Presidential Campaign", en Journalism Quarterly, 59, pp. 414-419.

MYLONAS, Yiannis (2012): "Media and the Economic Crisis of the EU: The 'Culturalization' of a Systemic Crisis and Bild Zeitung's Framing of Greece", en Triple C, 10 (2), 646-671.

NATIONAL COMMISSION ON THE CAUSES OF THE FINANCIAL AND ECONOMIC CRISIS IN THE UNITED STATES (2011): The Financial Crisis Inquiry Report. Final Report of the National Commission on the Causes of the Financial and Economic Crisis in the United States. Official Government Edition. January, 2011. http://www.gpo.gov/fdsys/pkg/GPO-FCIC/pdf/GPO-FCIC.pdf [consultado el 11/03/11]

OEI Oficina de Evaluación Independiente del FMI (2011): Desempeño del FMI en el periodo previo a la crisis financiera y económica: La supervisión del FMI entre 2004-07, 10 de enero. http://www.abc.es/gestordocumental/uploads/economia/InformeFMICrisis.pdf [consultado el 25/05/11]

PÉREZ OLIVA, Milagros (2012): “¿Contribuyen los medios a la crisis?”, en El País, 29 de enero.

PÉREZ-PEÑA, Ricardo (2008): “Amid Market Turmoil, Some Journalists Try to Tone Down Emotion", en The New York Times, September 22.

PESTON, Robert (2009): "In the new digital world. There is a stronger need than ever for subsidised, public-service news". Ethical Space: The International Journal of Communication Ethics, 6 (3/4), Special Issue, pp. 10-21.

QUIRING, Oliver y WEBER, Mathias (2012): "Between Usefulness and Legitimacy: Media Coverage of Governmental intervention during the Financial Crisis and Selected Effects", en The International Journal of Press/Politics, 17 (3), 294-315.

RAJAN, Raghuram G. (2010): Fault Lines. How Hidden Fractures Still Threaten the World Economy. Princeton: Princeton University Press.

ROUSH, Chris (2008): “Unheeded Warnings", en American Journalism Review, October/November, pp. 35-39.

ROUSH, Chris (2011): “The Financial Press: It’s Not as Bad as Its Reputation", en A. SCHIFFRIN. Bad News. How America's Business Press Missed the Story of the Century (pp. 54-70). New York, The New Press.

SANDVOSS, Cornel (2010): "Conceptualizing the Global Economic Crisis in Popular Communication Research", en Popular Communication, 8, 2010, 154-161. 
SARABIA-PANOL, Zeny \& SISON, Marianne D. (2010): "Who's to Blame for the GFC? Insights from Southsea Asia", en Journalism Studies, 11 (2), pp. 274-278.

SATIJA, Neena (2009): "Why Did Financial Journalists Miss the Financial Crisis", en Hufftington Post, April 22. http://www.huffingtonpost.com/neena-satija/post _304_b_190057.html [consultado el 6/6/2010]

SCHIFFERES, Steve y COULTER, Stephen (2012): "Downloading disaster: BBC news online coverage of the global financial crisis", en Journalism. Online First Version, June 14, 2012.

SCHIFFERES, Steve (2012): "Trust-meltdown for business journalism", en British Journalism Review, 23 (2), 55-59.

SCHIFFRIN, Anya (2011a): Bad News. How America's Business Press Missed the Story of the Century. New York: The New Press.

SCHIFFRIN, Anya (2011b): "The business press must prove its economic literacy", en The Guardian, 2 March.

SCHIFFRIN, Anya y FAGAN, Ryan (2012): "Are we all Keynesians now? The US Press and the America Recovery Act of 2009", en Journalism (Online First Version, Sep. 18, 2012).

SCHRANZ, Mario y EISENEGGER, Mark (2011): "The Media Construction of the Financial Crisis in a Comparative Perspective -An Analysis of Newspapers in the UK, USA and Switzerland between 2007 and 2009”, en Swiss Journal of Sociology, 37 (2), pp. 241-258.

SCHECHTER, Danny (2009): “Credit crisis: How did we miss it?", en British Journalism Review, 20 (1), pp. 19-26.

SPOSITO, Sean (2009): "Coverage of the meltdown: Did we blow it?". www.sabew.org. http://sabew.org/2009/08/coverage-of-the-meltdown-did-weblow-it/ [consultado el 2/01/2010]

STARKMAN, Dean (2009): "Power Problem", en Columbia Journalism Review, May/June, pp. 24-30.

STIGLITZ, Joseph E. (2011): "The Media and the Crisis: An Information Theoretic Approach", en A. SCHIFFRIN. Bad News. How America's Business Press Missed the Story of the Century (pp. 22-36). New York, The New Press.

STRUPP, Joe (2008): "Brauchli: Newspapers Warned Readers of Economic Crisis", en Editor\&Publisher, October 1.

http://www.editorandpublisher.com/ Headlines/Article/Brauchli-NewspapersWarned-Readers-of-Economic-Crisis [consultado el 8/08/11].

STULLER, Jay (2008): "The changing world of business journalism", en The Conference Board Review, July/August, 41-47.

TAMBINI, Damian (2010): “What are financial journalists for?”, en Journalism Studies, 11 (2), pp. 158-174. 
TAMBINI, Damian (2008): What is Financial Journalism For? Ethics and Responsibility in a Time of Crisis and Change. London, Polis-School of Economics.

THE ECONOMIST (2010): "Spain and the Anglo-Saxon press: Spain shoots the messenger", 9 February, 2010.

THOMPSON, Katherine (2008): "Reshaping the Financial Times' newsroom for the Credit Crisis", en editorsweblog.org, October 9.

THOMPSON, Peter A. (2009): "Market Manipulation? Applying the Propaganda Model to Financial Media Reporting", en Westminster Papers in Communication and Culture, 6 (2), 73-96.

TITLEY, Gavan (2012): "Budgetjam! A communications intervention in the political-economic crisis in Ireland”, en Journalism, Online First Version, June 14, 2012.

TRACY, James F. (2012): “Covering 'financial terrorism': The Greek debt crisis in the US news media", en Journalism Practice, 6 (4), 513-529.

TREMLETT, Giles (2010): “Anglo-Saxon media out to sink us, says Spain”, en The Guardian, 14 February 2010.

TULLOCH, John (2009): "From amnesia to apocalypse: Reflections on journalism and the credit crunch", en Ethical Space: The International Journal of Communication Ethics, 6 (3/4), Special Issue, 99-109.

UK PARLIAMENT TREASURY COMMITTEE (2009): "Role of press in banking crisis. Written evidence", House of Commons, UK, February 4.

USHER, Nikki (2012): "Ignored, uninterested, and the blame game: How the New York Times, Marketplace and TheStreet distanced themselves from preventing the 2007-2009 crisis", en Journalism (Online first version, September 13, 2012). 